\title{
LA ALQUIMIA DE LA ORIENTACION: PROPUESTA PEDAGOGICA DESDE EL PARADIGMA DE LA COMPLEJIDAD \\ COUNSELING'S ALCHEMY: PEDAGOGICAL PROPOSAL FROM \\ THE COMPLEXITY PARADIGM
}

\author{
SECCIÓN ESPECIAL \\ Volumen 15, Número 1 \\ Enero - Abril \\ pp. 1-19
}

Este número se publicó el $1^{\circ}$ de enero de 2015

DOI: $\underline{\mathrm{dx} . \text { doi.org/10.15517/aie.v15i1.17628 }}$

Silvia Alvarado Cordero

Revista indizada en REDALYC, SCIELO

Revista distribuida en las bases de datos:

CATÁLOGO DE LATINDEX, IRESIE, CLASE, DIALNET, DOAJ, E-REVIST@S, SHERPA/ROMEO, QUALIS, MIAR

Revista registrada en los directorios:

ULRICH'S, REDIE, RINACE, OEI, MAESTROTECA, PREAL, CLACSO 


\title{
LA ALQUIMIA DE LA ORIENTACION: PROPUESTA PEDAGOGICA DESDE EL PARADIGMA DE LA COMPLEJIDAD \\ COUNSELING'S ALCHEMY: PEDAGOGICAL PROPOSAL FROM \\ THE COMPLEXITY PARADIGM
}

\begin{abstract}
Silvia Alvarado Cordero ${ }^{1}$
Resumen: Este trabajo busca brindar un aporte a la disciplina de Orientación al proponer una forma de incorporar el paradigma de la complejidad al quehacer profesional. Se incluye una revisión de la injerencia de diferentes teorías en el desarrollo histórico de esta disciplina y se ejemplifica la aplicación de los principios de auto organización, interdependencia y sustentabilidad a la práctica de la Orientación. Sustentada en estos fundamentos teóricos, se diseñó e implementó una propuesta pedagógica en un centro educativo de Educación Primaria. Se realizó un trabajo de campo con 22 docentes y 380 estudiantes de una escuela pública en donde, durante un mes, se generaron espacios de participación y expresión creativa, con acciones a nivel de aula y de institución. En las acciones realizadas se subrayó constantemente la importancia de iniciar con el cambio individual en pequeños actos, como el ahorro energético, el manejo de desechos, la armonía en las relaciones interpersonales, se desplegó un abanico de posibilidades que emergió de las personas participantes. Los resultados y conclusiones evidencian importantes alcances y aportes que realiza la Orientación a la calidad de vida personal y social desde el contexto educativo, desde donde se convierte en una experiencia pedagógica exitosa, que puede ser homologada en otros contextos.
\end{abstract}

Palabras clave: ORIENTACIÓN, PARADIGMA DE LA COMPLEJIDAD, PROPUESTA PEDAGÓGICA, COSTA RICA.

Abstract: This paper aims a contribution in Counseling field suggesting how to incorporate the complexity paradigm to professional tasks. A review of the influence of several theories on the historical development of the discipline is included along with the application of the principles of selforganization, interdependence, and sustainability to the practice of Counseling is exemplified in the paper. Based on those theoretical foundations, a pedagogical proposal was designed and implemented in a primary school. Twenty-tow teachers and 380 from the public primary school participated in a variety of opportunities for creative expression, and activities in the classroom as well as in the institution as a whole. During field work, the importance of starting with individual change in small actions such as energy saving, waste management, harmony in interpersonal relationships, and an array of unimaginable possibilities that arose from the participants, was constantly stressed. Results and conclusions show that Counseling in the primary schools can deliver important inputs that contribute to a better personal and social quality of life, becoming a successful pedagogical experience that can be transferred to other contexts.

Keywords: COUNSELING, COMPLEXITY PARADIGM, PEDAGOGICAL PROPOSAL, COSTA RICA.

\footnotetext{
1 Profesora de la Escuela de Orientación y Educación Especial, Investigadora del Instituto de Investigación en Educación, ambos de la Universidad de Costa Rica.
}

Dirección electrónica: sil.alvarado@gmail.com

Documento recibido: 10 de junio, 2014

Enviado a corrección: 12 de agosto, 2014

Aprobado: $1^{\circ}$ de diciembre, 2014 


\section{Introducción}

Así como los Alquimistas desde la Edad Media invirtieron mucho esfuerzo en encontrar la "Piedra filosofal" (sustancia que según los creyentes en la alquimia tendría propiedades extraordinarias, como la capacidad de trasmutar los metales en oro), mi búsqueda de cómo contribuir con la disciplina de la Orientación, me ha llevado a descubrir que un camino puede ser mediante la incorporación de los planteamientos del paradigma de la complejidad.

En el contexto de la celebración de los 50 años de la Orientación en Costa Rica, se consideró importante retomar la presente propuesta pedagógica en Orientación, dada la pertinencia y relevancia de contar con insumos permitan reflexionar acerca de los aportes de los paradigmas y cómo influyen en el quehacer de la disciplina.

En el primer apartado se incluyen algunos planteamientos teóricos del desarrollo histórico de la Orientación, del paradigma de la complejidad o emergente y se plantea incorporar los principios de autoorganización, interdependencia y sustentabilidad a la práctica profesional. En el apartado de metodología se explica desde este posicionamiento teórico el diseño e implementación de una propuesta pedagógica, que se inicia con el planteamiento de ejes estratégicos y objetivos específicos, que incorporados a la práctica profesional en Orientación en un centro educativo de primaria, permitieron plantear los resultados y conclusiones del trabajo de campo realizado con la incorporación del paradigma de la complejidad.

\section{Acercamiento teórico}

\subsection{Una mirada a la historia de la Orientación}

En el desarrollo histórico de la humanidad se muestra que ha existido la necesidad y disposición de las personas a colaborarse mutuamente, en el seno de la sociedad algunas personas se han dedicado de manera particular a desarrollar esta acción. La Orientación se configura como una disciplina que busca contribuir al desarrollo y bienestar de las personas. En Costa Rica a lo largo de la historia, se ha evidenciado la influencia múltiples tendencias, entre ellas el paradigma positivista, la metodología experimental, el desarrollo de las técnicas estadísticas y psicométricas y la emergencia de la corriente de salud mental, así como de los movimientos sociales de reforma que culminaron con la implementación de la Orientación en el Sistema Educativo.

En la literatura relacionada con el desarrollo de la Orientación, se plantean dos fases: la precientífica y la científica, ambas se diferencian por los métodos y técnicas que en cada 
periodo se han utilizado. En la primera fase pre científica, que va desde tiempos antiguos hasta finales del siglo XIX, se considera que existía una permanente búsqueda de orientación de las personas, se ayudaba a los jóvenes a convertirse en miembros de la sociedad y a lidiar con las adversidades de lo cotidiano. En la segunda fase, denominada científica, es la época en la que se recurre a la verificación experimental para explicar los fenómenos humanos. De esta manera, la Orientación como ciencia tiene su origen en el razonamiento y la observación (Masis, Mendoza y Quesada, 1996).

Dentro de este desarrollo histórico-científico, la Orientación es considerada una disciplina, ya que estudia un área específica, es decir se interesa por el ser humano y su desarrollo. Además, posee un marco de referencia teórico y práctico, que aporta los conocimientos necesarios para dar significado a los procesos orientadores, así la Orientación evoluciona con el conocimiento científico y se caracteriza por los paradigmas empleados.

La elección de un paradigma científico determina el tipo de situaciones que se desean explorar, las técnicas por usar, las teorías por construir, así como la naturaleza y el valor de las contribuciones en el desarrollo del potencial humano. Por ejemplo, la Orientación a partir del desarrollo de la filosofía positivista, como enfoque epistemológico científico, pretendió cuantificar toda realidad humana, a este paradigma se le atribuye una visión del mundo positivista, hipotético-deductiva, particularista, objetiva y orientada hacia resultados, propio de la ciencia natural (Gordillo, 1988).

Con el paradigma fenomenológico, la Orientación se adscribe a una visión de mundo fenoménica, inductiva, holística, subjetiva y orientada hacia el propio proceso. Desde este paradigma, se retoma la importancia de adoptar una actitud de exploración y apertura, a fin de comprender mejor las situaciones, captando los eventos con el significado que tienen para la persona que los está viviendo.

La Orientación ha estructurado su teoría con base en una serie de ideas y conocimientos prácticos sobre fenómenos y hechos afines en la conducta habitual de las personas, por ello se considera una ciencia aplicada. Es decir, la persona orientadora utiliza los conocimientos básicos tanto propios como de diferentes disciplinas a fin de individualizar situaciones surgidas en los diferentes ámbitos de trabajo (Castro, Chaves y Morales, 1996).

La labor de la Orientación como campo profesional, surgió a mediados del siglo XX y en Costa Rica ha pasado por varios momentos. Entre 1948 y fines de 1964, surgen las primeras ideas de establecer la Orientación como servicio. Algunos de los factores que influyeron en el surgimiento son la necesidad de impulsar el crecimiento económico y social 
del país, el interés por instaurar una educación media que respondiera a las necesidades de la nación y los estudiantes, el deseo de incorporar al sistema educativo, técnicas y procedimientos de Orientación basados en la pedagogía, la psicología, la sociología y la higiene mental, así como la necesidad de ofrecer a los y las estudiantes orientación vocacional. En el año de 1964, con la Reforma a la Enseñanza Media, se establece oficialmente en Costa Rica el servicio de Orientación (Pereira, 2006).

Las personas que iniciaron en Costa Rica la labor orientadora fueron docentes de secundaria que recibieron un asesoramiento en técnicas y procedimientos específicos, se les llamaba inspectores, que más tarde pasaron a denominarse auxiliares de Orientación, aunque conservaban sus funciones de control que venían ejerciendo, se realiza el cambio de nombre al puesto al ampliar las funciones.

Dentro de este periodo, en marzo de 1964, comienza el plan regular para la preparación de profesionales en Orientación en la facultad de Educación en la Universidad de Costa Rica, tratando de responder a las necesidades del Sistema Educativo. En ese momento el énfasis de la Orientación era la ubicación vocacional de las personas, utilizando instrumentos psicométricos para evaluar características personales, las cuales predijeran el éxito vocacional o académico. Durante los 10 años siguientes, se establecen y expanden los servicios de Orientación, enfatizando en el logro de la adaptación del estudiante al medio educativo. En 1973, surge la necesidad de analizar los principios y fines de la Orientación, dentro de la propuesta del Plan Nacional de Desarrollo Educativo y de los fines de la Educación costarricense. Producto de ello, en 1975, se plantean los principios de la Orientación con una visión de ser humano como unidad biopsicosocial, tomando en cuenta las etapas evolutivas y las diferencias personales. Además se involucra a todo el personal de la institución educativa y a la familia como responsables del proceso orientador (Pereira, 2006).

En estos años se divide la Orientación, tanto para el estudio como para el ejercicio profesional en las áreas vocacional, educativa y personal social. Según la Universidad de Costa Rica (2000, p. 7):

la Orientación existe en Costa Rica como disciplina aplicada de las Ciencias de la Educación, porque se han dado ciertas condiciones históricas, socioeconómicas, políticas y culturales, que han hecho sentir la necesidad de ofrecer a la población estudiantil fundamentalmente, una atención especializada a sus necesidades de orientación personal, educacional y vocacional. 
El propósito debía dirigirse al logro académico y al éxito personal, como vía para la toma de decisiones vocacionales. En 1985, inició un énfasis en la práctica de la Orientación en prevención, al aplicar en el campo educativo, el enfoque denominado "Circulo del bienestar", el cual fue aprobado por el Consejo Superior de Educación en 1990 y se tomó un marco de referencia para la prevención de problemas psicosociales que pudiera presentar la población estudiantil. Este enfoque concibe al ser humano con una visión holística, formado por diferentes dimensiones: física, emocional, ética, espiritual, vocacional y social, además se incorporó la dimensión cognoscitiva como parte de esta visión integral (Baldares, 2004).

Con este aporte, se da un fortalecimiento del desarrollo personal de las orientadas y los orientados por medio del énfasis en el plan de vida y el desarrollo de las habilidades para enfrentar la vida responsable y armoniosamente. Poco a poco, las personas profesionales en Orientación logran abarcar otros ámbitos de trabajo además del educativo, entre ellos se puede mencionar el vocacional, familiar y el penitenciario, por lo que la identidad de la persona que realiza Orientación se ha expandido, al visualizarse en diferentes escenarios laborales.

En el devenir de la historia de Orientación como ciencia, disciplina y profesión se ha pasado por un proceso de transformación, que ha requerido que se adapte a los cambios del momento histórico-social en que se encontraba.

Actualmente, se considera que los procesos de Orientación ofrecen condiciones para favorecer el crecimiento, el desarrollo personal y vocacional, preparando a las personas para enfrentar las diferentes etapas y situaciones de la vida. Se considera que para lograrlo es importante estimular el autoconocimiento, la autonomía, la autoestima, la comprensión del medio, el juicio crítico y la construcción de una escala de valores reflexiva, que orienten la conducta hacia la superación y dirección personal, con un sentido ético (Pereira, 2006).

\subsection{La Orientación desde el paradigma de la complejidad}

La rapidez y profundidad de los cambios que suceden en la actualidad, en todas las dimensiones de la vida, ponen en evidencia que en el siglo XXI la historia de la humanidad ha entrado en una etapa sin precedentes y con imprevisibles repercusiones para las sociedades.

La ciencia revoluciona la materia, la vida y la mente en este siglo, con extraordinarios acontecimientos como la revolución informática, biomolecular y cuántica. Hemos pasado de una economía de mercado "nacionalista" a economías supra-mundiales, globalizadas en 
manos de unas cuantas personas, bajo la denominación de compañías anónimas. También hemos pasado de una concepción natural de la vida humana a la fertilización in vitro, de la información documental a una hipertextualización no solo escrita sino que también virtual.

El desarrollo vertiginoso y continuo avance de los medios de comunicación permite conocer y obtener la información de manera directa y en el momento, con imágenes y sonidos que pueden darnos una sensación de mayor veracidad sobre los acontecimientos sucedidos.

Además, observando el desarrollo de las relaciones interpersonales, con la naturaleza y el cosmos, se advierte una gran necesidad de solidaridad que no dependa de leyes, ni decretos, de imposiciones, o de anuncios publicitarios, sino una solidaridad que sea fruto de la iniciativa de cada persona, que sea expresión de la buena voluntad, que lleve a proponer, establecer y ejecutar acciones de bien común, cargadas de positivismo y del convencimiento propio de ser partícipe de la vida en su conjunto, por lo tanto, una vida plena de interrelacionalidad.

Desde finales del siglo XX y en lo que va del Siglo XXI el concepto de complejidad se ha integrado en muchos ámbitos, es una forma de abordar la realidad en las ciencias experimentales y que se extiende a las ciencias sociales. La teoría de los sistemas complejos es un modelo explicativo de los fenómenos del mundo que integra aportes de distintas ramas del conocimiento científico. El paradigma de la complejidad es una opción ideológica, un modelo de pensamiento y de acción ciudadana (Bonil, Sanmartí, Tomás y Pujol, 2004).

Según Rodríguez y Aguirre (2011), la complejidad puede entenderse, como un paradigma científico emergente que involucra un nuevo modo de hacer y entender la ciencia, extendiendo los límites y criterios de cientificidad, más allá de las fronteras de la ciencia moderna, ancladas sobre los principios rectores del mecanicismo, el reduccionismo y el determinismo.

Las primeras referencias al paradigma de la complejidad las plantea Edgar Morin (s.f.), en contraposición a lo que denomina paradigma de la simplificación, propone la necesidad de construir un pensamiento complejo y la importancia de una acción ciudadana orientada por una forma de posicionarse en el mundo.

El pensamiento de Morin (s.f.) aborda el conocimiento como un proceso que es a la vez, biológico, cerebral, espiritual, lógico, lingüístico, cultural, social e histórico, mientras que la epistemología tradicional asume el conocimiento sólo desde el punto de vista cognitivo. 
Este nuevo planteamiento tiene enormes consecuencias en el planteamiento de las ciencias, la educación, la cultura, la sociedad, al plantear que la complejidad es, el tejido de eventos, acciones, interacciones, determinaciones, azares, que constituyen nuestro mundo fenoménico.

A lo largo de las últimas décadas el paradigma de la complejidad ha sido tomado como referente desde distintos ámbitos, en especial desde aquellos asociados a la educación ambiental y principalmente en países latinoamericanos, como una respuesta a los problemas sociales y ambientales actuales. El paradigma de la complejidad supone una opción ideológica de valores, pensamiento y acción, reúne aportaciones de campos muy diversos que configuran una perspectiva ética, de construcción del conocimiento y una perspectiva de la acción (Bonil et al., 2004).

La Orientación, es una disciplina joven y el desarrollo histórico que ha tenido en Costa Rica, es parte de su constitución. En el encuentro con el Paradigma de la complejidad se evidencia que sus planteamientos se encuentran en sintonía con los fundamentos de la Orientación, ya que las personas necesitan descubrir su camino y es ahí donde la Orientación puede estimular en el individuo su desarrollo, el cual emergerá del descubrimiento de su interrelacionalidad.

Los procesos de cambio en la calidad de vida personal y social, así como en los procesos generados desde la práctica de la Orientación se pueden lograr con esta nueva visión del paradigma emergente, ya que permite una transformación trascendental de las reflexiones, valores y formas de visualizar y actuar en el mundo, desde la totalidad de dichas interrelaciones.

Cabe mencionar que también Boff (2001), insiste que el reto para la humanidad depende de la capacidad que tengamos de asumir el desafío frente a los nuevos modos de ser, de sentir, de pensar, de valorar, de actuar, de rezar, que necesariamente llevan a nuevos valores, sueños y comportamientos asumidos por un número cada vez mayor de personas y comunidades.

La vida se presenta para el ser humano como un proceso creativo que él mismo debe desarrollar mediante la autoorganización para enfrentar las diferentes situaciones que se le presentan. Actualmente, la Orientación como disciplina enfoca sus esfuerzos no solo a personas o grupos en situación de riesgo, sino a la población total, porque su misión no es "remediar" problemas, su accionar pretende el fortalecimiento de las habilidades y potencialidades de las personas para que logren su desarrollo y del entorno. Esta dimensión 
es un gran paso que permite que la Orientación se abra a la totalidad de la vida, porque desde el paradigma de la complejidad la persona es considerada parte de la interrelacionalidad con otras personas, la ecología y el cosmos.

Por lo tanto, se hace cada vez más necesario la incorporación de una forma amplia de entender al ser humano, si la Orientación lo visualiza desde el paradigma de la complejidad, se comprende que la persona genera su bienestar desde el conjunto de la vida, de la totalidad y más allá de estos vínculos desde la unidad de la vida. Dicha unidad implica ser este todo y a la vez conservar la especificidad que caracteriza a cada ser vivo.

Maturana (1999) plantea que los sistemas auto-producidos (una célula, un organismo, la conciencia o psique, una corporación, entre otros) constan de una red de procesos u operaciones, que pueden transformar o destruir componentes pero en los que el mismo sistema opera su identidad y la mantiene a través de esa red de procesos de interacción entre sus elementos, correspondiendo al principio de autopoiésis, la cual se considera una propiedad básica de los seres vivos, puesto que son sistemas determinados en su estructura, es decir, son sistemas tales que cuando algo externo incide sobre ellos, los efectos dependen de ellos mismos, de su estructura en ese instante y no de lo externo.

La Orientación puede trabajar con personas asumiendo el principio de la autopoiésis, es decir, en los procesos que realiza apoyar la capacidad que poseen las personas de autoproducir y auto-reproducir, así si se estimula el bienestar y genera desde su interior la búsqueda y logro del mismo. Por lo tanto, es una la necesidad visualizar a los seres humanos como organismos integrales, en los aspectos positivos del comportamiento humano: la felicidad, la satisfacción, la alegría, la serenidad, la diversión y el éxtasis, el crecimiento personal y en la autorrealización (Capra, 1982).

Además, la Orientación puede retomar el paradigma de la complejidad, al incorporar en su práctica los principios de autoorganización, interdependencia y sustentabilidad, como evidencia que la vida se caracteriza por ser un proceso que se gestiona a sí mismo, llevando así a trasformar el cómo se han realizado la labor orientadora y que le he denominado lograr la alquimia de la Orientación.

\subsection{Principio de autoorganización}

Los sistemas naturales y sociales en el desarrollo de la vida son flujos permanentes de autoorganización, dejando atrás al modelo que enfatizó en el orden establecido, la uniformidad, el control extremo, la estabilidad, pasando a ver la realidad cotidiana desde una 
visión caracterizada por lo dinámico, divergente, incierto, que responde a una variedad de posibilidades. El ser humano, es autoorganización en “... un proceso en el que se construye a sí mismo, no mediante adaptación a un entorno rígido, pues este también es dinámico, vivo, en una continua co evolución..." (Alvarado, 2006, p. 66).

Si se visualiza la Orientación en su quehacer cotidiano desde este principio, se alienta a permanentemente repensar las propuestas y flexibilizar sus procesos, para favorecer la creatividad y el desarrollo de la vida en el equilibrio dinámico, logrando así colaborar en la gestación de un modelo educativo autoorganizado, de coconstrucción, en donde todos y todas tenemos la capacidad de regenerar el tejido de la vida que nos une. Este principio llama a que las personas pasen de ser seres a los que hay que ayudarles a resolver sus problemas a ser personas generadoras de sus propias vidas.

Al trabajar y compartir con familias que viven en condiciones de pobreza extrema, se empezó a visualizar la aplicabilidad del paradigma a la labor orientadora, constantemente advertía en sus experiencias la posibilidad que tenemos los seres vivos de autoorganizarnos y propiciar continuos cambios en respuesta al medio, además de la posibilidad de permanecer en continua adaptación, aprendizaje y desarrollo, los cuales se manifiestan como las características clave del comportamiento de los seres vivos.

El potencial que tiene la autoorganización es el de facilitar y promover ambientes diversos que permitan la emergencia y desarrollo de las habilidades del ser vivo en plenitud y desde una complejidad incalculable de posibilidades de desarrollo. Por ejemplo, cuando se realizan procesos de orientación colectiva o con pequeños grupos es una premisa establecer en conjunto las normas de convivencia, por lo que en muchas oportunidades se convierten en imposiciones cargadas de "debemos". Frente a esto la Orientación puede abrirse a la alquimia o trasformación al brindar espacios para la autoorganización de las personas, propiciando acciones concretas para que emerjan desde ellas mismas los lazos de convivencia. Este principio nos llama a confiar en la capacidad de las personas para auto organizarse.

El incorporar el principio de autoorganización también implica la posibilidad de encontrar oleadas de pensamientos y recrear la convivencia a partir de la reconstrucción de lo existente. Somos sistemas adaptativos, donde la autoorganización tiene un papel central en el desarrollo de posibilidades vitales, nuevas, creativas e inspiradoras para el desarrollo. 


\subsection{Principio de interdependencia}

El otro principio clave para comprender los procesos de realización humana, es la interdependencia, que evidencia que todo está conectado en una misma red. El éxito de los procesos vitales dependerá del tipo de interrelaciones que logremos establecer entre los diferentes elementos que conformamos el todo, ya que estamos interconectados en una vasta y compleja red. Establecer esta visión de totalidad de la vida, implica conocer los elementos bioquímicos que configuran nuestro organismo, las relaciones diarias que conforman nuestra cultura, las diferentes organizaciones sociales, evidenciando la diversidad en la totalidad y viceversa, para dejar de pensar fragmentariamente.

Asumir el principio de interdependencia en la labor orientadora insta a incorporar la visión sistémica para establecer redes tomando en cuenta su contexto cotidiano, que permiten conocer y establecer acciones a favor del bienestar de los seres humanos. Cuando se apoya a docentes en la búsqueda de alternativas resulta muy significativo prestar atención a todos los detalles, un ejemplo fue una situación en un centro educativo, cuando una docente solicita apoyo a Orientación expresando sentimientos de enojo, preocupación e impotencia por una niña de 9 años, quien mostraba desinterés hacia el estudio, descuido personal y malos hábitos de higiene y que no respondía a sus solicitudes, de cambiar dichos hábitos. Después de una escucha activa y empática con la maestra, se estableció en conjunto las posibles acciones, el primer paso fue visitar su hogar en el precario, conversar con ella y su familia. Este inicio fue muy significativo tanto para la docente como para la estudiante, quienes iniciaron una relación más estrecha al sentirse comprendida en sus particularidades. En esta situación fue muy perceptible la interrelacionalidad, ya que toda la familia evidenciaba los mismos hábitos y para generar un cambio, fue necesario realizarlo desde las posibilidades de las personas adultas responsables de la niña, tanto por la salud individual como por la convivencia con otras personas.

De esta manera, el comportamiento de una persona no puede retomarse aislado de su contexto y de las interrelaciones que establece, orientar es un acto total que no puede ser fragmentado, es un proceso que implica muchos niveles de la conciencia humana, como el afectivo, el físico, el social y el espiritual rebasando por completo lo puramente cognitivo (Gallegos, 1999). 


\subsection{Principio de sustentabilidad}

Los principios de autoorganización e interdependencia se complementan con el principio de sustentabilidad y viceversa. Este aspecto revela la posibilidad que tiene cada organismo, cada especie, cada sistema, de conservar su estabilidad. "La sustentabilidad tiene en cuenta el equilibrio dinámico y autorregulador vigente en la naturaleza gracias a la cadena de interdependencias y complementariedades entre todos los seres, especialmente los vivos, que viven de recursos permanentemente reciclados y por ello indefinidamente sostenibles..." (Boff, 2001, p. 131).

De esta manera, la Orientación también puede contribuir a la construcción de la civilización planetaria, en donde todos somos parte de un solo tejido que nos une a pesar de nuestra diversidad. El desarrollo de una nueva cultura de sustentabilidad, implica la transformación de la manera de ver, sentir, enfocar y vivir nuestras relaciones con el planeta Tierra y con las demás personas. Para lograr la trasformación de condiciones adversas a condiciones de prosperidad y bienestar.

De esta manera para lograr la Alquimia de la Orientación, al incorporar el paradigma de la complejidad, es necesario que los procesos que se desarrollen no sean rígidos, apegados a normativas o procedimientos administrativos. Se requiere conocer la interrelacionalidad asociada a cada particularidad de las personas, establecer relaciones de empatía auténtica, caracterizadas por lazos de fraternidad, equidad y solidaridad.

Además, la educación implica “...una búsqueda permanente de un equilibrio dinámico entre la libertad individual y la responsabilidad social" (Alvarado, 2006, p. 122), en la misma sintonía, la Orientación puede generar núcleos de solidaridad que provoquen verdaderos encuentros fraternos entre los seres vivos, el planeta y el cosmos; en donde los seres humanos, como miembros de una sociedad, desarrollemos una red de procesos, actos, encuentros, conductas y emociones que configuren un sistema de convivencia y éste penetre en todos los aspectos del vivir, en todas la dimensiones del hacer y emocionar.

Desde ésta óptica, la Orientación como disciplina, en sincronía con la educación, es un agente no sólo de renovación interna y de cambio social, porque va más allá del aprender las ideas o los hechos que aparecen en los documentos; es también aprender de la vida, de la sonrisa de un niño, del jugueteo de los cachorros, en el juego de las olas del mar, en el trinar de los pájaros, al contemplar la puesta de sol, al observar la naturaleza con sus múltiples colores y formas, a partir de este aprendizaje, se desarrolla un sentimiento de responsabilidad hacia la Tierra y un compromiso con la vida que hay en ella. 
Necesitamos una Orientación capaz de propiciar espacios donde la persona pueda observar y descubrir por sí misma el significado de la totalidad. Se requiere, un trabajo creativo, que fortalezca las condiciones para la construcción de una sociedad inclusiva. Además, se puede promover el desarrollo de la innovación, la alteridad, de cambio ante las circunstancias adversas y de búsqueda de consenso con las demás personas.

Se requiere que la práctica de la Orientación contribuya a contemplar los problemas ambientales y del desarrollo en su globalidad, teniendo en cuenta las repercusiones a corto, mediano y largo plazo, tanto para la humanidad como para el planeta, para constituirse en un instrumento eficaz para que la humanidad pueda progresar hacia los ideales de paz, libertad y justicia. Contribuyendo de esta manera al desarrollo humano, para que sea más armonioso, genuino y disminuir la exclusión.

Lo ecológico no debe ser un tema más con una metodología específica, sino que su singularidad reside en su transversalidad, en el relacionar todas las experiencias y todas las formas de comprensión por ser complementarias y útiles para nuestro conocimiento del universo y nuestra funcionalidad en él.

Realizar Orientación incorporando el principio de sustentabilidad, implica ser coherentes con nuestras acciones cotidianas, así como diseñar y desarrollar estrategias alternativas a la devastación ecológica, la pauperización y la exclusión de las personas. La aplicación de estos principios se ejemplifica en el siguiente trabajo de campo a favor de la vida.

\section{Aspectos metodológicos}

Desde el paradigma de la complejidad, cabe mencionar que la metodología no es una receta que se siguió paso a paso de manera lineal, ya que los múltiples caminos para abordar la propuesta pedagógica llevaron a un proceso de construcción en conjunto con las personas participantes. En concordancia con los principios del paradigma de la complejidad, durante la aplicación se respetó la diversidad humana, los contextos y los procesos recursivos de la producción del conocimiento. Así, el primer momento fue establecer en conjunto ejes estratégicos y objetivos específicos que se plasmaron posteriormente en acciones que permitieron el desarrollo del pensamiento, la observación y análisis desde el paradigma de la complejidad.

La propuesta pedagógica consistió en realizar Orientación, fundamentada en los principios de la autoorganización, interdependencia y sustentabilidad, con el objetivo de 
proporcionar a las personas participantes una mayor sensibilidad ética y estética del Universo y del ser humano de manera armoniosa.

\section{Participantes y contexto}

El trabajo de campo se realizó con 22 docentes y 380 estudiantes de I y II ciclo de una escuela de atención prioritaria, que se caracteriza por condiciones de precariedad y pobreza estructural que viven las comunidades de las cuales procede el estudiantado.

En este ámbito educativo, se tomó en cuenta que durante el mes de junio existen fechas especiales que en se realiza alguna celebración, entre ellas: la Semana de la cultura de Paz, Semana del Ambiente, Día de la Música, Semana Nacional de la Orientación y Semana Nacional Sí a la Vida. Por lo que se generó un proceso sinérgico de participación, creatividad, expresividad y relacionalidad; los saberes se crearon y recrearon en la vida cotidiana.

\section{Ejes estratégicos}

Para el planteamiento de las acciones la premisa inicial fue plantear con los y las participantes el contenido de los ejes estratégicos que guiaron todas las iniciativas, donde cada uno se convirtió a su vez en etapas de trabajo. A su vez en cada etapa se volvían a retomar todos los ejes, según como se muestra en la siguiente figura 1, están relacionados de manera dinámica y recurrente, es decir no hay un orden preestablecido.

Figura 1

Ejes estratégicos para propuesta pedagógica

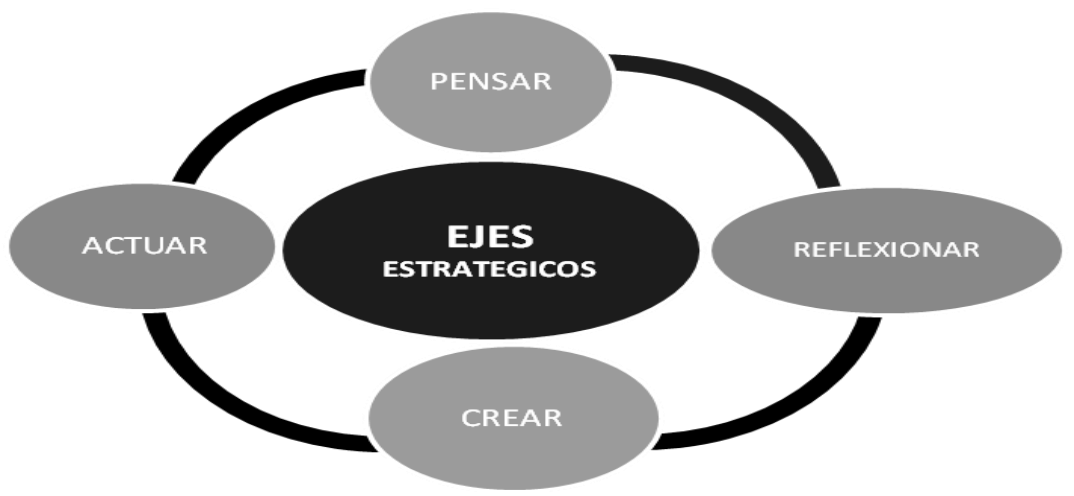

Fuente: Elaboración propia 
Una vez establecidos los ejes estratégicos, se procedió a plantear objetivos específicos, los cuales son la guía articuladora de la propuesta pedagógica; las acciones fueron generadas desde la acción orientadora en conjunto con estudiantes, docentes y familias. Por lo tanto, las acciones planteadas respondían a las necesidades y características específicas de la población participante.

\section{Resultados y análisis}

La implementación de la propuesta pedagógica inició con encuentros fraternos en pequeños subgrupos donde todas las personas participantes tenían pensamientos y sentimientos importantes que compartir, así desde un espacio de intercambio fraterno se inició con una pequeña acción, que poco a poco se fue ampliando, involucrando a muchas personas, que respondieron con entusiasmo y compromiso, con la conciencia de que somos capaces de readquirir nuestra reverencia por la vida, de reemplazar el rumbo de la historia para vencer con el deseo de cooperar e incrementar los lazos de comunión entre los grupos, para hacer de la vida humana, la celebración gozosa que supone el proceso permanente de aprendizaje y transformación a favor de la vida.

Fundamentados en los ejes estratégicos planteados, se formularon preguntas clave y objetivos específicos que fueron la guía para las iniciativas de las personas participantes con respecto a las acciones que deseaban realizar, según se muestra en la siguiente tabla 1:

Tabla 1 Propuesta pedagógica: Construyendo una ciudadanía ambiental desde Orientación

\begin{tabular}{l|ll}
\multicolumn{1}{c|}{ Etapas } & \multicolumn{1}{c}{ Objetivos específicos } \\
\hline $\begin{array}{l}\text { 1. ¿Qué siento? } \\
\text { Nuestras vivencias de } \\
\text { interrelacionalidad }\end{array}$ & 1.1 Identificar las preocupaciones, sentimientos y experiencias \\
relacionadas con el desarrollo sustentable.
\end{tabular}


Durante un mes se generaron espacios de participación y expresión creativa, con acciones a nivel de aula y de institución. Se inició con la organización de espacios de reflexión acerca de la cultura de sustentabilidad con el personal docente, fue realmente sorprendente el encontrar puntos de congruencia y análisis, se expresaron sentimientos, preocupaciones y propuestas. Se evidenció el principio de autoorganización que subraya el potencial que tiene de facilitar y promover ambientes diversos que permitan la emergencia y desarrollo de las habilidades del ser vivo en plenitud y desde una complejidad incalculable de posibilidades de desarrollo.

Resultó muy acertado el abordar, desde las vivencias con las docentes y después con el estudiantado los enunciados de La Carta de la Tierra, que ofrece un conjunto de valores organizados en 16 principios para la construcción de un mundo mejor. Este es un recurso valioso que aporta una visión holística e integrada de los problemas sociales y ambientales de la humanidad, considera a la ecología como un nuevo paradigma para relacionarse con la naturaleza, presenta que todos los seres estamos conectados entre sí formando un sistema inmenso y complejo, coincidiendo con lo planteado en el principios de interdependencia que nos insta a dejar la costumbre de pensar fragmentariamente.

Cabe mencionar que el principio de la carta de la Tierra que más fue seleccionado por los y las estudiantes, fue el No. 1 "Respeto la Tierra y la Vida en todas sus manifestaciones". El estudiantado lo presentó creativamente con dramatizaciones, carteles, canciones y hasta bailes, compartieron sus reflexiones y propuestas, entre ellas, no botar basura en el aula, hacer basureros con cajitas para cada pupitre, no pelear, respetar a sus compañeros y compañeras, cuidar a los animales, sembrar plantas, entre otros, acorde con lo planteado en el principio de sustentabilidad, que menciona que el desarrollo de una nueva cultura de sustentabilidad, implica transformación en la manera de ver, sentir, enfocar y vivir nuestras relaciones con el planeta Tierra, con las demás personas.

Tanto del personal docente como del estudiantado, surgieron varias iniciativas por ejemplo, se realizó un mural educativo de manera que todas las semanas se le agregaban los elementos nuevos, que se iban conociendo y acciones que se realizaban, así de un árbol solo con unas ramas, se le incluyeron muchísimas flores coloreadas por el estudiantado, luego se fueron agregando mensajes detallados inspirados en los principios de la Carta de la Tierra. Otras acciones realizadas que se pueden mencionar son dramatizaciones para los actos cívicos, talleres de expresión creativa con materiales de desecho, talleres participativos en las aulas, dibujos libres, montajes de recortes, exposición de trabajos, video foros con las 
películas el Milagro de la Mariposa Azul y la Carta a la Tierra, así como redacciones y trabajos extractase.

Se evidenció que se logó generar un clima institucional de solidaridad, entusiasmo y creatividad, logrando así que la práctica de la Orientación colabore a contemplar los problemas ambientales y del desarrollo en su globalidad, teniendo en cuenta las repercusiones a corto, mediano y largo plazo, tanto para la humanidad como para el planeta, para constituirse en un instrumento eficaz para poder progresar hacia los ideales de paz, libertad y justicia.

De las acciones realizadas se subrayaba constantemente la importancia de iniciar con el cambio individual en pequeños actos, como el ahorro energético, el manejo de desechos, la armonía en las relaciones interpersonales, en fin, se desplegó un abanico de posibilidades inimaginables y que surgieron de las propias personas participantes. Se generó un proceso autoorganizado, en el que los niños y niñas que se movilizan con sus amistades, familiares, la comunidad y hasta el comercio para compartir sus aprendizajes y contribuir en la recolección de materiales. Se conformó un club ambientalista de 30 estudiantes, integrado por un niño y una niña de cada grupo, para que junto a cada docente propiciaran continuar la "campaña" en pro de la vida en el planeta, cumpliendo así el objetivo de generar iniciativas que favorezcan la cultura de la sustentabilidad.

De manera sincrónica, las maestras del Comité de Aseo y Ornato se ofrecieron voluntariamente para iniciar un proyecto de reciclaje a nivel institucional tomando en cuenta a los y las estudiantes y sus familias. Surgieron muchas iniciativas para el embellecimiento externo de la institución, con campañas de limpieza, colocación de plantas y pintura de la escuela. Además se inició con recolección de papel blanco, periódico y botellas plásticas, se colocaron bolsas rotuladas en cada aula y se consiguieron estañones. Dadas las características de la escuela, es un gran logro, que en un mes se haya recolectado casi 600 kilos de periódico. Con esta respuestas de las personas participantes se muestra lo planteado en los aspectos teóricos, que la Orientación como disciplina, en sincronía con la educación, es un agente no sólo de renovación interna y de cambio social, porque va más allá del aprender las ideas o los hechos que aparecen en los documentos, es también aprender de la vida.

Con esta experiencia pedagógica se evidencia que los procesos de cambio en la calidad de vida personal y social, así como en los procesos generados desde la práctica de la Orientación se pueden lograr con esta nueva visión del paradigma, ya que permite una 
transformación trascendental de las reflexiones, valores y formas de visualizar y actuar en el mundo desde la totalidad de dichas interrelaciones. Coincidiendo con lo planteado por Morin (s.f) la complejidad es relación y apertura, sin un punto de vista único y absoluto desde el cual abordar la realidad y el conocimiento, donde es esencial considerar a las personas de manera integral junto con su contexto. Por lo tanto, la labor orientadora no es para responder a una normativa o un lineamiento laboral, todo se realiza con la conciencia de que la Orientación es un agente de trasformación que contribuye a generar La Vida.

\section{Conclusiones}

La Alquimia de la Orientación desde el Paradigma Emergente, es un gran desafío que configura un mapa actualizado de las posibles aplicaciones al despliegue de la Vida para contribuir en la constitución de una Nueva Humanidad, no se refiere a una ilusión o sentimentalismo, evoca a que toda acción debe ser intencionada y fundamentada para generar interrelaciones en pro de la Vida.

La práctica de la Orientación en el ámbito educativo, permite a la persona profesional en Orientación ser creativa y estar en continua transformación. Cada profesional mediante sus acciones realizadas desde el paradigma de la complejidad puede irradiar a otras personas esta nueva visión de la vida para contribuir así en los ideales de paz, libertad y justicia.

La Orientación se despliega desde la vida cotidiana teniendo presente la interrelacionalidad inherente a todo en la Vida, asumiendo la posibilidad que tiene la persona de lograr la autoorganización para propiciar el surgimiento de acciones creativas, con las cuales puede afrontar las diferentes vicisitudes del diario vivir de manera armoniosa y sustentable.

En el ámbito educativo, la Orientación pasa de ser una disciplina que interviene en determinadas situaciones de vida a ser generadora de procesos alquímicos a favor del incremento de la cantidad y calidad de acciones positivas individuales y sociales. Ello, además puede incidir en la prevención de los comportamientos destructivos, contribuir en la educación emocional y el clima social en que se desenvuelven dichas personas.

La Orientación desde esta nueva visión paradigmática implica nuevos desafíos, el desarrollo de una práctica en donde se promuevan transformaciones desde la persona para que pueda asumir las situaciones adversas transformándolas a positivo. 
Los nuevos cometidos de la Orientación serán un continuo dinámico, creativo y esperanzador no solo para aquellos que conforman el ámbito educativo, sino que también para la sociedad en general. Por lo tanto, la redefinición de la disciplina de la Orientación, inicia con la transformación interior, coherente, e intencionada de cada persona.

La alquimia se inicia desde la propia vida de la persona que ejerce la Orientación, teniendo la capacidad de trasladar los procesos de conocimiento básicos hacia una nueva conciencia, hacia toda la humanidad. Durante esta experiencia se logró religar sentimientos y saberes, para ir perfilando la Orientación desde el paradigma de la complejidad y despojarse de la visión mecanicista de la vida y del estilo tradicional de construcción del aprendizaje. Para lograr este cometido se requiere de un camino donde los valores de justicia, bondad, belleza y felicidad, estén presentes. Es a la vez una posibilidad de construir una Nueva Humanidad, la cual se logrará con nuestra vida, en las actividades diarias y desde el ejercicio profesional de la Orientación, que en sincronía con la Educación es propiciadora de procesos de trasformación.

\section{Referencias}

Alvarado, Eladio. (2006). Pasos hacia otra epistemología. Costa Rica: Librería Alma Mater.

Baldares, Thelma. (2004). Cuarenta años de Orientación en el Sistema Educativo Costarricense [Foro]. San José, Costa Rica: Paraninfo de la UNED.

Bonil, Josep, Sanmartí, Neus, Tomás, Catalina y Pujol, Rosa María. (2004). Un nuevo marco para orientar respuestas a las dinámicas sociales: el paradigma de la complejidad. Investigación en la Escuela, (53), 1-20. Recuperado de: http://www7.uc.cl/sw educ/educacion/grecia/plano/html/pdfs/linea investigacion/Educa cion Ambiental IEA/IEA 004.pdf

Boff, Leonardo. (2001). Espiritualidad un camino de transformación. Río de Janeiro, Brasil: Editorial Sextante.

Capra, Fritjof. (1982). El Punto crucial: ciencia, sociedad y cultura naciente. Buenos Aires, Argentina: Editorial Troquel.

Castro, Dalia, Chaves, Inés y Morales, Yolanda. (1996). La Orientación en Costa Rica: un análisis histórico sobre el desarrollo teórico-metodológico. (Tesis de licenciatura). Escuela de Orientación y Educación Especial, Facultad de Educación, Universidad de Costa Rica, San José, Costa Rica.

Gallegos, Ramón. (1999). Educación Holista: Pedagogía del amor. México: Ed. Universal.

Gordillo, María. (1988). La Orientación en el proceso educativo. Pamplona, España: Ediciones Universidad de Navarra. 
Maturana, Humberto. (1999). Transformación en la convivencia. Santiago de Chile: Ediciones Dolmen.

Morin, Edgar. (s.f). Introducción al pensamiento complejo. Recuperado de: http://www.edgarmorin.org/images/publicaciones/edgar-morin-introduccion-alpensamiento-complejo.pdf

Pereira, Teresita. (2006). Mediación Docente de la Orientación Educativa. Costa Rica: EUNED.

Rodríguez, Leonardo y Aguirre, Julio. (2011). Teorías de la complejidad y ciencias sociales Nuevas Estrategias Epistemológicas y Metodológicas. Nómadas. Revista Crítica de Ciencias Sociales y Jurídicas, 30(2), 1-20. Recuperado de: http://pendientedemigracion.ucm.es/info/nomadas/30/rdzzoya aguirre.pdf

Universidad de Costa Rica. (2000). Plan de estudios, bachillerato y licenciatura en Ciencias de la Información con énfasis en Orientación. San José, Costa Rica: Escuela de Orientación y Educación Especial. 